Journal of Advanced College of Engineering and Management, Vol. 6, 2021

\title{
RIESZ POTENTIAL AND FRACTIONAL MAXIMAL FUNCTION
}

\author{
Santosh Ghimire \\ Department of Applied Sciences and Chemical Engineering, Institute of Engineering, \\ Pulchowk Campus, Tribhuvan University,Nepal \\ Email Address: santoshghimire@ioe.edu.np
}

\begin{abstract}
:
In this article, we begin with Riesz potential. We then discuss some properties of the Riesz potential. Finally we discuss a relation of Riesz Potential with fractional maximal function in the sense that fractional maximal function can be controlled by Riesz potential and the fractional maximal function maps the space $L^{p}$ to $L^{q}$ whenever the Riesz potential does.
\end{abstract}

Keywords: Riesz Potential, Schwartz class, Fractional Maximal function.

\section{Introduction}

Riesz Potential is a potential in mathematics which is named after the Hungarian Mathematician Marcel Riesz. Let $\mathrm{s}$ be a complex number whose real part is greater than zero. Then Riesz Potential of order $\mathrm{s}$ is denoted by $I_{S}$ and is defined as $I_{S}=(-\Delta)^{\frac{-s}{2}}$ where $\Delta$ is Laplacian operator given by $\Delta=\partial_{1}^{2}+\cdots+$ $\partial_{n}^{2}$.

Moreover, the Riesz Potential $I_{S}$, associated with a locally integrable function $\mathrm{f}$ on $R^{n}$ is defined as:

$$
I_{S}(f)=2^{-s} \pi^{\frac{-n}{2}} \frac{\Gamma\left(\frac{n-s}{2}\right)}{\Gamma\left(\frac{s}{2}\right)} \int_{R^{n}} f(x-y)|y|^{-n+s} d y
$$

We note that the above singular integral is well defined if $\mathrm{f}$ decays sufficiently rapidly at infinity.Thus the above integral is convergent if the function $f$ is in the Schwartz class where the Schwartz Class, roughly speaking, is the collection of smooth function whose all of the derivatives decay faster than the reciprocal of any polynomial at infinity.

More generally the Riesz potential can be defined in a weak sense as the convolution $I_{s}(f)=f * K_{S}$ where $K_{S}$ is the locally integrable function given by

$$
K_{s}(x)=2^{-s} \pi^{\frac{-n}{2}} \frac{\Gamma\left(\frac{n-s}{2}\right)}{\Gamma\left(\frac{s}{2}\right)} \frac{1}{|x|^{n-s}} .
$$

Readers are suggested to refer [3] for more about Riesz potential.

With the introduction of maximal functions one can better understand the concept of averages of functions inanalysis. Maximal functions are widely used in differentiation theory in analysis. Roughly the maximal function is defined as the largest value of the averages of functions over all possible balls which contain a fixed point. Maximal functions appear in many forms. The most important of these is the Hardy-Littlewood maximal function. We now define Hardy-Little wood maximal function:

Let $f \in L^{p}\left(\mathbb{R}^{n}\right), 1 \leq p<\infty$. Let 


$$
M f(x)=\sup _{r>0} \frac{1}{|B(x, r)|} \int_{B(x, r)}|f(y)| d y
$$

Then $M f$ is called the Hardy-Littlewood maximal function of $f$. In the definition ofmaximal function, all the balls are centered at the point $x$ Therefore, this is centered Hardy-Littlewoodmaximal function. Similarly, we can define uncentered Hardy-Littlewood maximal as:

$$
M^{u} f(x)=\sup _{r>0, x \in B} \frac{1}{|B|} \int_{B}|f(y)| d y
$$

One can easily show that the centered and uncentered H-L maximal functions are equivalent in the sense that value of these functions is controlled by each other up to some constant. Moreover, the Hardy-Little wood maximal functions can also be defined using cubes in $\mathbb{R}^{n}$.

For $0 \leq s<n$, one can define another maximal function as:

$$
M^{s} f(x)=\sup _{t>0} \frac{1}{\left(v_{n} t^{n}\right)^{\frac{n-s}{n}}} \int_{|y| \leq t}|f(x-y)| d y
$$

where $v_{n}$ is the volume of the unit ball in $\mathbb{R}^{n}$. Due to the fraction power $\frac{n-s}{n}$, this maximal function is called fractional maximal function. Readers are suggested to refer [3] and [2] for more about these maximal functions.

The fractional maximal function and Riesz potential are related in various ways. Adams [1] showed that:

2. Theorem: Let $s>0,1<p<\frac{n}{s}, 1 \leq q<\infty$ such that $\frac{1}{r}=\frac{1}{p}-\frac{s}{n}+\frac{s p}{n q}$. Then there exists a constant $C>0$ (depending on the previous parameters) such that for all positive functions $f$ we have

G.V. Welland [4] showed that:

$$
\left\|I_{S}(f)\right\|_{L^{r}}=C\left\|M^{\frac{n}{p}}(f)\right\|_{L^{q}}^{\frac{s p}{n}}\|f\|_{L^{p}}^{1-\frac{s p}{n p}} .
$$

3. Theorem: Let $0<\alpha<n$ and suppose $0<\varepsilon<\min (\alpha, n-\alpha)$. Then there exists a constant depending only on $\alpha, \varepsilon$ and $\mathrm{n}$ such that for all compactly supported bounded function $\mathrm{f}$ we have

$$
\left|I_{s(f)}\right|=C \sqrt{M^{\alpha-\varepsilon}(f) M^{\alpha+\varepsilon}(f)} .
$$

Next, we discuss some properties related to Riesz potential.

(1) Let $0<s, t<\square$ be such that $s+t<n$. Then Riesz potential satisfies $I_{s} I_{t}=I_{s+t}$. This property is called semi group property. For this, consider a function $f \in \mathbb{S}\left(\mathbb{R}^{n}\right)$ where $\mathbb{S}\left(\mathbb{R}^{n}\right)$ denotes Schwartz class. Then by definition, we have

$$
\widehat{I_{s+t}}(f)(\xi)=(-\Delta)^{-\widehat{\left(\frac{s+t}{2}\right)}}(f)(\xi)=(2 \pi|\xi|)^{-(s+t)} \hat{f}(\xi)
$$

where the symbol $\hat{f}$ denotes the Fourier transform of $f$. Again,

$$
\begin{aligned}
I_{s}\left(\widehat{I_{t}(f)}\right)(\xi)=(-\Delta)^{-\widehat{\left(\frac{s}{2}\right)}} I_{t}(f)(\xi) & \\
& =(2 \pi|\xi|)^{-s} \widehat{I_{t}(f)}(\xi) \\
& =(2 \pi|\xi|)^{-s}(2 \pi|\xi|)^{-t} \hat{f}(\xi) \\
& =(2 \pi|\xi|)^{-(s+t)} \hat{f}(\xi)
\end{aligned}
$$

Therefore we have $\widehat{I_{s+t}}(f)=I_{s}\left(\widehat{I_{t}(f)}\right)$ for all functions $\in \mathbb{S}\left(\mathbb{R}^{n}\right)$. This gives $I_{s} I_{t}=I_{s+t}$. This proves the semigroup property. We now discuss another property.

(2)Riesz potential satisfies the following operator identity:

whenever $\operatorname{Re} s>2 \operatorname{Re} z$.

$$
I_{s}(-\Delta)^{z}=(-\Delta)^{z} I_{s}=I_{s-2 z}=(-\Delta)^{z-\frac{s}{2}}
$$


For this, we have $\operatorname{Re}(s-2 z)>0$. Then

$$
\begin{gathered}
\widehat{I_{s-2 z}}(f)(\xi)=(-\Delta)^{\left.-\sqrt{\frac{s-2 z}{2}}\right)}(f)(\xi) \\
=(2 \pi|\xi|)^{-(s-2 z)} \hat{f}(\xi) \\
=(2 \pi|\xi|)^{-s+2 z} \hat{f}(\xi) \\
=(2 \pi|\xi|)^{2 z-s} \hat{f}(\xi)
\end{gathered}
$$

Clearly we have

$$
(-\Delta)^{\widehat{z-\frac{s}{2}}}(f)(\xi)=(2 \pi|\xi|)^{2 z-s} \hat{f}(\xi)
$$

Again we have

$$
\begin{aligned}
I_{s}\left(\widehat{(-\Delta)^{z}}(f)\right)(\xi) & =(2 \pi|\xi|)^{-s}\left(\widehat{-\Delta)^{z}}(f)(\xi)\right. \\
& =(2 \pi|\xi|)^{-s}(2 \pi|\xi|)^{2 z} \hat{f}(\xi) \\
& =(2 \pi|\xi|)^{2 z-s} \hat{f}(\xi) .
\end{aligned}
$$

Similarly we can show:

$$
\left(\overline{-\Delta)^{z}} I_{s}(f)(\xi)=(2 \pi|\xi|)^{2 z-s} \hat{f}(\xi) .\right.
$$

So we have

$$
\begin{gathered}
\widehat{I_{S-2 z}}(f)(\xi)=(-\Delta)^{\widehat{z-\frac{s}{2}}}(f)(\xi) \\
=I_{S}\left(\widehat{(-\Delta)^{z}}(f)\right)(\xi) \\
=\left(\widehat{-\Delta)^{z}} I_{S}(f)(\xi) .\right.
\end{gathered}
$$

This gives

$$
I_{S}(-\Delta)^{z}=(-\Delta)^{z} I_{S}=I_{S-2 z}=(-\Delta)^{z-\frac{s}{2}}
$$

(3) For all complex number $z$, we have $\left\langle(-\square)^{z}(f) \mid(-\square)^{z}(g)\right\rangle=\langle f \mid g\rangle$ whenever the Fourier transforms of $\mathrm{f}$ and $\mathrm{g}$ vanish to sufficiently high order at the origin and the symbol $\langle\mathrm{f} \mid \mathrm{g}\rangle$ denotes the complex inner product.

$$
\begin{aligned}
\left\langle(-\Delta)^{z}(f) \mid(-\Delta)^{z}(g)\right\rangle & =\int(-\Delta)^{z}(f)(x) \overline{(-\Delta)^{z} g(x)} d x \\
& =\int \overline{(-\Delta)^{z}}(f)(\xi) \overline{(-\Delta)^{z} g(\xi)} d \xi \\
& =\int(2 \pi|\xi|)^{2 z} \hat{f}(\xi)(2 \pi|\xi|)^{-2 z} \overline{\hat{g}}(\xi) d \xi \\
& =\int f(x) \overline{g(x)} d x \\
& =\langle\mathrm{f} \mid \mathrm{g}\rangle .
\end{aligned}
$$


We now discuss another property of Riesz potential.

(4) Given Re $s>0$, then we find a complex number $\alpha$ such that $\left\langle I_{s}(f) \mid f\right\rangle=\|\left(-\widehat{\square)^{\alpha}}(f) \|_{L^{2}}^{2}\right.$ whenever Fourier transform of $f$ vanishes to sufficiently high order at origin. For this, we have

$$
\begin{gathered}
\int\left(I_{s} f\right)(x) \overline{f(x)} d x=\|\left(-\overline{\Delta)^{\alpha}}(f) \|_{L^{2}}^{2}\right. \\
\int \widehat{I_{s} f}(\xi) \overline{\overline{f(\xi)}} d \xi=\int\left|\left(-\Delta \widehat{)^{\alpha}(f)}\right)(\xi)\right|^{2} d \xi \\
\int(2 \pi|\xi|)^{-s} \hat{f}(\xi) \overline{\overline{f(\xi)}} d \xi=\int\left|(2 \pi|\xi|)^{-2 \alpha} \hat{f}(\xi)\right|^{2} d \xi \\
\int(2 \pi|\xi|)^{-s}|\hat{f}(\xi)|^{2} d \xi=\int\left((2 \pi|\xi|)^{-4 \alpha}\right)|\hat{f}(\xi)|^{2} d \xi
\end{gathered}
$$

This gives:

$$
\int\left[(2 \pi|\xi|)^{-s}-(2 \pi|\xi|)^{-4 \alpha}\right]|\hat{f}(\xi)|^{2} d \xi=0 .
$$

This is possible only when we have $(2 \pi|\xi|)^{-s}-(2 \pi|\xi|)^{-4 \alpha}=0$ i.e. $(2 \pi|\xi|)^{-s}=(2 \pi|\xi|)^{-4 \alpha}$. Thus we have $4 \alpha=s$. Hence we get $\alpha=\frac{s}{4}$.

Finally we establish the following result related to fractional maximal function and Riesz potential:

For some constant $C$ we have $M^{s}(f) \leq C I_{s}(f)$ for all $f \geq 0$. Moreover, the fractional maximal function $M^{S}$ maps the space $L^{p}$ to $L^{q}$ whenever the Riesz potential $I_{S}$ does.

For $f \geq 0, I_{s}(f)(x)=C(n, s) \int_{R^{n}} f(x-y)|y|^{-n+s} d y$ where $C(n, s)=2^{-s} \pi^{\frac{-n}{2}} \frac{\Gamma\left(\frac{n-s}{2}\right)}{\Gamma\left(\frac{s}{2}\right)}$.

For $|y| \leq t$ and $0 \leq s<n$ it follows that $1 \leq\left(\frac{t}{|y|}\right)^{n-s}=t^{n-s}|y|^{s-n}$. Therefore

$$
\begin{aligned}
\frac{1}{\left(v_{n} t^{n}\right)^{\frac{n-s}{n}}} \int_{|y| \leq t}|f(x-y)| d y & \leq \frac{1}{\left(v_{n} t^{n}\right)^{\frac{n-s}{n}}} \int_{|y| \leq t}|f(x-y)| t^{n-s}|y|^{s-n} d y \\
& =\frac{1}{\left(v_{n}\right)^{\frac{n-s}{n}}} I_{s}(f)(x) \\
& =C I_{s}(f)(x)
\end{aligned}
$$

where $C=\frac{1}{\left(v_{n}\right)^{\frac{n-s}{n}}}$. Now taking supremum over all $t>0$, we have $M^{s}(f)(x) \leq C I_{s}(f)(x)$ for every $x$ and for every $f \geq 0$. Therefore $M^{s}(f) \leq C I_{s}(f)$ for every $f \geq 0$.

Next suppose that $I_{S}$ maps $L^{p}$ to $L^{q}$. One can easily show that for some constant $\mathrm{C},\left\|I_{S}(f)\right\|_{L^{q}} \leq$ $C\|f\|_{L^{p}}$ for $f \in L^{p}$. Now for any $f \in L^{p}$ we have

$$
\begin{aligned}
\left\|M_{S}(f)\right\|_{L^{q}} & =\left(\int\left|M^{s}(f)(x)\right|^{p} d x\right)^{\frac{1}{p}} \\
& \leq\left(\int\left|C I_{S}(f)(x)\right|^{p} d x\right)^{\frac{1}{p}} \\
& =C\left\|I_{S}(f)\right\|_{L^{p}} \\
& \leq C^{\prime}\|f\|_{L^{q}} .
\end{aligned}
$$

This proves that the fractional maximal function $M^{s}$ maps the space $L^{p}$ to space $L^{q}$ whenever Riesz potential does. This proves the result. 
4. Conclusion: We discussed some properties of Riesz potential with their proof. Moreover, we also related Riesz potential with the fractional maximal function.

\section{References:}

1) Adams, D.(1975), A note on Riesz potential, Duke Mathematics Journal, Volume 42, page 765-778.

2) Banuelos R. and Moore C.N.(1999), Probabilistic Behavior of Harmonic Functions, Birkhauser Verlag

3) Grafakos L. (2009), Modern Fourier Analysis, Second edition, Springer.

4) Welland G.V. (1975), Weighted norm inequalities for fractional integrals, Proceeding of American Mathematical Society, Volume 51, page 143-148. 